\title{
Snippets: Tools for teaching on two levels
}

\author{
Anna Jarrett* \\ Eleanor Mann School of Nursing, University of Arkansas, Fayetteville, Arkansas, USA
}

Received: February 13, 2019

Accepted: July 14, 2019

Online Published: July 25, 2019

DOI: $10.5430 /$ jnep.v9n10p87

URL: https://doi.org/10.5430/jnep.v9n10p87

\begin{abstract}
There are times when the opportunity to create a novel approach to teaching presents itself. This is true of the following innovative approach to teaching difficult concepts using a dual level method to enhance comprehension by undergraduate nursing students. The purpose of this case study was to use short video recordings called Snippets, which were previously recorded by the instructor as a new two-level learning activity to augment nursing students' grasp of difficult concepts in a conventional didactic classroom, and to determine whether there was a difference in student satisfaction between didactic real-time presentations and didactic recorded 15-to-30 minute sessions with faculty present in the classroom. A mixed methods approach was used. Although there was no statistically significant difference in students' satisfaction between the two methods of lecture as reported by survey methods, content analysis supported using Snippets as an 'accessible' strategy for teaching on two levels to enhance learning. It is useful teaching strategy available to faculty in conventional nursing programs to augment learning for particularly difficult conceptual material.
\end{abstract}

Key Words: Snippets, Technology, Didactic, Recordings, Learning strategies, Two-level learning

\section{INTRODUCTION}

Nursing programs are increasing enrollments due to pressures to produce more graduates without adding additional faculty, in light of reduced financial and clinical resources. ${ }^{[1,2]}$ Schools of nursing must meet these demands and continue providing quality educational programs to produce competent nurses for their communities. Enrollment has increased four-fold in some undergraduate nursing programs. Many programs have used the cohort model to facilitate student matriculation through the nursing courses of study. The cohort model purports a community experience for students to provide support, encouragement, and comfort. $^{[3]}$ In addition, the nonnursing student now sits in the classroom with various undergraduate or graduate degrees but is still a novice to terms, and understanding of the nursing process. ${ }^{[4]}$
With this demand, classes that typically contained no more than 25 students in a small, quiet environment are now held in large arenas that can accommodate large numbers of students. For faculty, this can mean a more distant relationship with their students and can be a dis-satisfier for faculty. For example, faculty may or may not be able to identify each student by name. They will invariably know the stellar students and the struggling students, but the 'typically average' student may get lost in the mass.

Creative approaches to stimulate learning in an educationally sound environment have been ongoing over the past decade. Some strategies and models implemented include such things as broadcasting simulation case studies to the didactic classroom, engaging students through the use of classroom response systems commonly known as clickers, and embracing the flipped classroom pedagogical model for

\footnotetext{
*Correspondence: Anna Jarrett; Email: ajarrett@uark.edu; Address: Eleanor Mann School of Nursing, 606 North Razorback Road, Office 254, Fayetteville, Arkansas, USA.
} 
graduate nursing programs. ${ }^{[5-7]}$

In higher education, online enrollment for courses and programs of study exceeds the traditional graduate student population. In 2010, the United States online tertiary enrollment boasted a $17 \%$ growth rate, as compared to traditional higher education student population growth rate at $1.2 \%$. Graduate programs have responded to this consumer demand by developing innovative ways to reduce time and distance barriers to tertiary education. ${ }^{[8]}$

Undergraduate education will surely follow suit. Blended or hybrid course design has become a standard practice in undergraduate nursing programs in the forms of enabling, enhancing, or transforming blends. The conventional design of a blended course is one that integrates both online with face-to-face instruction in a planned, pedagogically valid manner. ${ }^{[9]}$

A Snippet may be defined as a small piece of something, or a component of something larger. It has been used in more specific contexts to refer to a sampling of music, a short piece of computer source code, or as a verb to solder into small pieces. ${ }^{[10]}$ For this study, the term Snippet has been re-defined as a small, specialized piece of knowledge that augments text, lecture, and tests in a conventional classroom. Nurse educators continue to discover ways to enhance both online and real time classroom learning. This effective use of Snippets and technology may enhance the classroom experience.

\section{Case presentation}

For this study, four Snippets were created for an undergraduate, first semester nursing pathophysiology class. All students had successfully completed anatomy and physiology prior to admission to the pathophysiology course. The Snippets were 15-30 minute video taped sessions by the faculty member at a time when not in the classroom delivering a conventional lecture. Slides and spreadsheets were used to conduct exercises to illustrate the procedure to correctly interpret findings. Presentations were pre-recorded before class in an environment conducive to uninterrupted concentration using readily available resources. Those presentations were: rhythm strip analysis and interpretations of complete blood count, electrocardiogram, and pulmonary function tests. Each recording was rehearsed once, recorded, and edited for length of presentation. The snippet was then delivered during class as part of the content for specific lectures, i.e., while lecturing on the respiratory pathology and diseases, $\mathrm{ABG}$ and then pulmonary function test interpretation test snippets were shown at the end of the system lecture. Hardcopy worksheets were distributed to the students to inter- pret the findings along with the pre-recorded Snippets. The faculty member had the opportunity to clarify statements, stop the recording as needed, and edit speech errors if they occurred.

The course was held in a computer laboratory twice weekly on Mondays and Thursday. On Mondays faculty presented the structure and function of each body system and augmented the lecture with one of the four Snippets for these three divisions of topics by body systems: cardiac, respiratory and hematology. The faculty member was always present in the classroom to augment discussion or answer statements each week throughout the course on both days of the week.

The structure and function lecture was selected because it is vitally important that one does not misspeak while explaining the pathophysiologic process under discussion, and it is important to connect theoretic knowledge with clinical practice. For example, when presenting content on the function of the cardiovascular system, textbooks may not demonstrate the relationship between action potentials and how it is reflected on an electrocardiogram. It is important to close that knowledge gap so nursing students can assimilate what they have learned in classroom with their experience in clinical environments.

Pre-recorded events with editing capability eliminate potential for making errors in content delivery. The class was arranged, so that the pathophysiology lecture was delivered in two 45-minute sessions with a 10-minute break between lectures. The Snippet was introduced each time at the end of the session to assist students to assimilate the information delivered. On Thursdays faculty presented the pathophysiology of diseases and disease processes related to the body system presented on Monday. Again on Thursdays, faculty was present in the classroom to augment discussion or answer questions each week. The lectures were delivered in two 45-minute sessions with a 10 -minute break between lectures, but Snippets were not used in that class session.

To measure student satisfaction, previously established course surveys methods were used to determine if students would comment on a preference for methods used to deliver course content. Because Likert scales consist of constrained data, non-parametric Fischer's Exact Test was used to analyze the aggregate responses to determine statistical significance between recorded and didactic lectures to determine if online delivery of this content is preferable to standard didactic lectures. To further understand students' thoughts and preferences, interviews were conducted to identify major themes in student feedback. 


\subsection{Methods}

The design for this small study used a mixed method approach using quantitative method (survey), and follow-up qualitative interviews. Inclusion criteria developed for the study included all nursing students enrolled in an upper class pathophysiology course which was taught by the faculty member conducting the study. Exclusion criteria were nursing students enrolled in higher level or another section of the same course in the same nursing program. The sample size was small, but adequate for survey methods. There were 41 students included in this study.

Rationale for choosing this course was the complexity and difficulty students experienced in particular areas of lecture in previous semesters and year. Although pathophysiology is a foundational course, it contains difficult concepts for nursing students to grasp without clinical experience on which to aid their memories.

\subsection{Survey}

The faculty member created a short Likert-scale item survey via the class's complementary web-enhanced interactive learning module. The survey with six statements was distributed to 41 students. The first two statements were grouped together to indicate usage of the Snippets in the classroom and as a study tool, while the remaining three statements were primarily posed to determine satisfaction with the didactic lecture content, to compare non-Snippet lectures with Snippet lectures. The first two statements used a 4-point Likert scale with $1=$ none; $2=$ some; $3=$ most; 4 $=$ all. The next three statements were rated using a 4-point Likert scale ranging from $1=$ not satisfied; $2=$ somewhat satisfied; $3=$ satisfied, and $4=$ very satisfied. The last statement inquired about a preference for Monday lectures over Thursday lectures. The listed responses to the last statement were not a true Likert Scale. However, they listed these possible responses: $1=$ preferred Monday lectures over Thursday lectures (with an explanation NOT to respond based on time course was taught); 2 = preferred Thursday lectures over Monday lectures; 3 = preferred Snippets over regular lecture, and $4=$ no preference of lectures. Statements were posed as seen in Figure 1.

1. The number of Snippets I watched
2. The number of Snippets recordings I used as additional
study tool for this course
3. My satisfaction with the use of Snippets as learning tools
4. My satisfaction with Monday lectures
5. My satisfaction with Thursday lectures
6. My preference of lectures day and style

Figure 1. Snippet survey

\section{FindingS}

To maintain confidentiality, data were de-identified by an anonymous web-based survey, thus protecting student names, identification numbers, or groups they were assigned. Demographics were limited to age and gender. The 41 students' data were inspected for missing information consistent with the inclusion criteria. There was one missing dataset from the survey. Statement \#3, how satisfied were you with the snippets had one response left blank. The group consisted was $95.12 \%$ female $(n=39)$ and $4.88 \%$ male $(n=2)$. The mean age of the sample was $23.4, \mathrm{SD}=7.56$ years.

The first two items inquired about the number of Snippets they watched, and whether they utilized them as a study tool for the course. Thirty-seven percent of the 41 students reported they had watched all of the Snippets, while 59\% reported they watched most Snippets. Because they were shown in class, that was not an unexpected response. A review of the class roster reflected an average of at least $90 \%$ attendance of the students across the 16-week semester. There was no penalty for missing class or points assigned for attendance, so the pressure and responsibility of learning the material was evident from the higher than expected attendance rate.

The second statement inquired about using Snippets as study tools. Ten percent of the students reported they used them when they had to miss class. Reasons for missing class were nursing organization research day, one soccer game in a distant community, and one student had a surgical intervention requiring a one-week absence. Thirty-seven percent responded that they never missed class. In addition, $49 \%$ responded they used the Snippets to study difficult material. Overall satisfaction with the Snippets revealed $50 \%$ of the students were very satisfied, and $38 \%$ were satisfied. Three percent preferred the Snippets over regular lectures.

Sixty-seven percent of the students responded that they were very satisfied with the quality of Monday lectures, $32 \%$ reported they were satisfied. There were no dis-satisfied students. Sixty-nine percent of the students responded they were very satisfied with Thursday lectures, 30\% reported they were satisfied, and again there were no dis-satisfied students. When asked to select their satisfaction with Monday lectures vs. Thursday lectures $60.9 \%$ reported they did not have a preference, and again when asked to select their satisfaction with Thursday lectures vs. Monday lectures, $59.8 \%$ cited no preference, which yielded adequately reliable responses.

After a review of each item was complete, statistical analysis of the responses was performed. Descriptive statistics (mean, standard deviation, standard error, range and confidence in- 
tervals) were measured. Nonparametric Fischer Exact Test compared numbers of responses on the three satisfaction scales: items \#3, \#4, and \#5 on the survey. No significant difference existed between statements $p=.89$ with an established level of significance $<.05$.

This exploratory study sought to determine if students preferred the use of Snippets over regular didactic lecture. A paired $t$-test was calculated to detect differences between student satisfaction with Monday lectures vs. Thursday lectures, $p=.06$ with alpha established at $<.05$. Students appeared to have no preference to lectures using Snippets to lectures without Snippets.

Because the study failed to show statistically significant difference between lectures in the classroom with or without the use Snippets, a review of the same faculty's end-of-course evaluation were compared to the previous semester where the faculty used similar class lectures but without the use of Snippets. The surveys were given to students via the complementary online hybrid course. A one-tail $t$-test was done to determine statistically significant differences between spring and fall course and faculty evaluation scores. Course scores returned $p=.12$; faculty scores returned $p=.27$ with an established level of significance alpha $<.05$. Although there was no statistical difference between course or faculty evaluation scores for the same pathophysiology, the overall scores were higher for both course and faculty for fall compared to spring.

Following the survey but prior to the results being reviewed, the students were asked to share their opinion about the use of the Snippets in the classroom. Group and individual comments were welcomed in an open forum session immediately following the course's final examination. Student responses were recorded and transcribed after the session. Content analysis was conducted to identify themes and move the essence of the interviews from a lower level of abstraction via the content, to a higher level of abstraction to find an overarching theme. ${ }^{[11]}$

Student feedback was coded and condensed into meaning units, then categorized into emerging themes. The overarching theme was 'quality' which prevailed in several statements. Lectures presented prior to the Snippets introduction were high quality, so it didn't matter if they were pre-recorded or not.

A second theme was 'faculty presence'. Students enjoyed the sessions with the faculty member present while the recording was ongoing. Snippets had the added benefit of faculty member presence with ability to stop the lecture to ask questions or clarify difficult concepts without interrupting the flow of the content delivery, not typically done in pure didactic lecture.

The last theme emerging was 'accessibility' for this group of students. These recording were viewed as valuable because they were made available throughout their nursing program, as well as for future faculty teaching pathophysiology in other sections. Students were satisfied with the use of Snippets, and they primarily used the Snippets as additional study tools for difficult material.

The following spring semester, this faculty member was asked to provide a 2-day Clinical Nurse Specialists review course for five recent graduates from an online graduate nursing program. The request was made by the Program Coordinator to record each session while lecturing to archive for future students' review prior to sitting for national certification examinations. Two days' prior to the course review session, the faculty member was notified that the computer laboratory was not available so real-time recording was not possible.

Because of the faculty member's previous experience using Snippets, the lectures were recorded for the first days' session prior to the class. The entire day's lectures were presented via recording. Faculty was present in the room, and the recordings were stopped multiple times to answer student questions and to clarify difficult concepts. The next day, the computer laboratory was available, so the second days' lectures were delivered live while being recorded. Because of the need to protect the privacy of each student, they were asked to hold their questions until the recording was complete.

Emerging themes from a small group interview initiated by the faculty member at the end of the review course on the second day were 'interrupt ability' and 'accessibility'. Because these students had a very recent comparison of pre-recorded and concurrent recording, the analysis varied slightly because the faculty member was present in the room both days, but unable to respond to questions immediately while recording concurrently. The first day's lecture methods using the videos while faculty was present with the ability to stop, clarify, back-up, or move faster through the content based on students' needs was the major benefit to these students. The second theme emerging was 'accessibility'. They voiced satisfaction with the recordings and were particularly grateful to use them as future study aids.

\section{Discussion}

After reviewing both sets of student interviews, the central theme emerging for both groups of students was 'accessibility'. The fact students could 'go back' and listen or rework 
the exercises was an enhancement to this course. It may be useful for faculty members to consider pre-recording Snippets and providing them as study guides as students' progress in academe.

Perhaps Snippets have a valuable place in didactic classrooms in undergraduate and graduate nursing programs to augment difficult material and to give students opportunities to stop the session and clarify concepts during lecture. No longer will students tolerate course instructors who begin their lectures with, "please hold your questions until after the lecture".

The study was limited to one baccalaureate nursing program with a modest sample size. Drawing predictive conclusions from such a small sample limits generalizability to the greater population. Snippets need to be tested using more sophisticated research designs with a larger sample size across disciplines. Although the quantitative findings were not of statistical significance, qualitative analysis strongly suggests the use of Snippets may be a valuable adjunct to classroom lectures.

A replication of the study is recommended using a larger sample size over multiple baccalaureate and graduate nursing programs with more sophisticated measurement methods. Perhaps a scoring system to score components of each lecture and each Snippet immediately after each presentation would provide a clearer preference and identify potential benefits of class-augmented Snippets. The use of Snippets could also be used as remediation tools with test pass rates measured after remediation. This may warrant further study as well.

\section{Conclusion}

The purpose of this study was to introduce the use of Snippets as a modality for faculty to augment and enhance learning in a controlled environment after a real time lecture occurred and to determine whether there was a difference in student satisfaction between didactic real-time presentations and didactic recorded 15-to-30 minute sessions with faculty present in the classroom while the recorded session was presented. The survey revealed there was no statistically significant difference between students' satisfaction of Monday lectures vs. Thursday lectures, but the major emerging theme from student interviews revealed the 'accessibility' of Snippets was a highly valued benefit for students. Snippets may well provide a significant advantage for use by faculty when presenting complex and difficult concepts in classroom settings.

\section{ACKNOWLEDGEMENTS}

I wish to thank the students who graciously participated in this pilot study and gave pertinent feedback about the usefulness of this type of two-level learning strategy.

\section{CONFlicts OF INTEREST Disclosure}

The author declares that there is no conflict of interest.

\section{REFERENCES}

[1] Siler B, DeBasio N, Roberts K. PROFILE of non-nurse college graduates enrolled in accelerated baccalaureate curricula: Results of a national study. Nursing Education Perspectives. 2008; 29(6): 336341.

[2] Institute of Medicine (IOM) Committee on the Robert Wood Johnson Foundation Initiative on the Future of Nursing, at the Institute of Medicine. The Future of Nursing: Leading Change, Advancing Health. Washington (DC): National Academies Press (US); 2011. I. The Future of Nursing Education.

[3] Raush D, Crawford E. Cohorts, communities of inquiry, and course delivery methods: UTC best practices in learning - The Hybrid Learning Community Model. The Journal of Continuing Higher Education. 2012; 60(3): 175-180. https://doi.org/10.1080/07377363.2 013.722428

[4] Jackson M, Marchi N. Graduate-entry education for nonnurses: preparation, pathways, and progress. Nursing Education Perspectives. 2019. PMid:31206416 https://doi.org/10.1097/01.N EP. 0000000000000510

[5] Critz C, Knight D. Using the flipped classroom in graduate nursing education. Nurse Educator. 2013; 38(5): 210-213. PMid:23969751 https://doi.org/10.1097/NNE.0b013e3182a0e56a
[6] Kalmakis K, Cunningham H, Lamoureux E, et al. Broadcasting simulation case studies to the didactic classroom. Nurse Educator. 2010; 35(6): 264-267. PMid:20975415 https : //doi .org/10.1097/NN E. 0b013e3181f7f $1 \mathrm{af}$

[7] Heaslip G, Donovan P, Cullen JG. Student response systems and learner engagement in large classes. Learning in Higher Education. 2013; 15(1): 11-24. https ://doi.org/10.1177/146978741351 4648

[8] Strand H, Fox-Young S, Long P, et al. A pilot project in distance education: Nurse practitioner students' experience of personal video capture technology as an assessment method of clinical skills. Nurse Education Today. 2013; 33: 253-257. PMid:22154874 https://doi.org/10.1016/j.nedt.2011.11.014

[9] McGee P, Reis A. Blended course design: A synthesis of best practices. Journal of Asynchronous Learning Networks. 2012; 16(4): 7-21. https://doi.org/10.24059/olj.v16i4.239

[10] Merriam Webster. Definition of snippet. Merriam-Webster: An Encyclopedia Britannica Company. 2019. Available from: http: //www .merriam-webster.com/thesaurus/snippet

[11] Erlingsson P, Brysiewicz C. A hands-on guide to doing content analysis. African Journal of Emergency Medicine. 2017; 7: 93-99. PMid:30456117 https://doi.org/10.1016/j . af jem.2017.0 8.001 